\title{
List of English names for Russian names
}

\section{Russian name}

Bastion 1

Bastion 2

Bastion 3

Bastion 4

Bastion 5

Bastion 6

Kornilov Bastion

Seleginsk Redoubt

Volhynia Redoubt

Pavlosky Point

Aleksandrovsky Battery

Mikhaelevsky Battery

Nikholaevsky Battery

\section{English name}

There does not appear to be an English name for this bastion

Little Redan

(Great) Redan

Flagstaff or Garden Battery

Central Battery

No English name

Malakhov

White Works

White Works

Paul Battery or Fort Paul

Alexander Battery or Fort Alexander

Michael Battery or Fort Michael

Nicholas Battery or Fort Nicholas 
Carol Helmstadter - 9781526140524 Downloaded from manchesterhive.com at $04 / 26 / 2023$ 12:32: $02 \mathrm{PM}$ 